\title{
Gesamtbetriebsräte: Neue Zuständigkeiten und die Folgen für betriebliche Arbeitsbeziehungen
}

\author{
Martin Behrens/Jürgen Kädtler
}

Gesamtbetriebsräte gelten zu Recht als durchsetzungsstarke Akteure der industriellen Beziehungen. Trotz ihrer vergleichsweise großen Bedeutung gibt es bis heute nur sehr wenige wissenschaftliche Arbeiten, die ihre Stellung zwischen Gewerkschaften und Betriebsräten genauer analysieren. Mit Rückgriff auf die Daten der WSI-Befragung der Gesamtbetriebsräte zur Restrukturierung (2006) wird die Arbeitsteilung zwischen den unterschiedlichen Betriebsratsebenen näher analysiert. Welche Folgen für eine effektive Vertretung der Belegschaftsinteressen ergeben sich aus der vorhandenen Tendenz, dass Gesamtbetriebsräte immer mehr Aufgabenfelder übernehmen?

\section{Gesamtbetriebsräte - Diskrepanz zwischen Recht und Praxis?}

Die Rechtsprechung zur Zuständigkeitsverteilung zwischen unterschiedlichen Betriebsratsebenen füllt Bände. Ohne die recht komplexe arbeitsrechtliche Auseinandersetzung hier im Detail wiedergeben zu können, lässt sich der aktuelle Stand der Gesetzgebung und Rechtsanwendung wie folgt zusammenfassen: Bei der Verteilung von Kompetenzen und Ressourcen zwischen örtlichem Betriebsrat (BR) und Gesamtbetriebsrat (GBR) hat der örtliche Betriebsrat eindeutig die Nase vorn. Die originäre Zuständigkeit einer anderen Ebene als die des örtlichen Betriebsrates ist demnach nur dann gegeben, wenn sich eine überbetriebliche Regelung als „zwingend erforderlich" erweist (Britz 2006, S. 131). Jedoch können die örtlichen Betriebsräte in Mehrbetriebsunternehmen die Regelung von Gegenständen von allgemeiner Bedeutung auf die Ebene des Gesamtbetriebsrats übertragen ( $\$ 50$ II BetrVG).

Tatsächlich kamen schon in der Vergangenheit immer wieder Zweifel daran auf, ob die gelebte Praxis mit dem geltenden Recht übereinstimmt. So stellte Rancke bereits vor 25 Jahren fest, dass eine „massierte Entscheidungszuständigkeit des Gesamtbetriebsrates" bestehe (Rancke 1982, S. 351) und thematisierte den daraus entstehenden Konflikt zwischen „dem Geltungsanspruch betriebsverfassungsrechtlicher Normen und der abweichenden betrieblichen Mitbestimmungspraxis" (ebd., S. 250). Auch neuere empirische Befunde deuten an, dass sich an dieser Situation we- nig geändert hat. So kommt eine Untersuchung von Hoffmann (2005) für den Bereich der Automobilindustrie zu dem Schluss, dass sich zwischen örtlichen Betriebsräten und Gesamtbetriebsräten ein hierarchisches Verhältnis herauskristallisiert hat. Demnach beklagt ein beachtlicher Teil der befragten örtlichen Betriebsräte eine Verlagerung der Entscheidungskompetenzen hin zum Gesamtbetriebsrat. Auch Wassermann berichtet über eine Aufwertung des GBRs zulasten der örtlichen Betriebsräte und führt diese auf fortschreitende Zentralisierungsprozesse auf der Managementseite zurück (Wassermann 2002, S. 145; Wassermann/Rudolph 2005, S. 74f).

\subsection{BR UND GBR IM DUALEN SYSTEM DER ARBEITNEHMER- VERTRETUNG}

Ob sich der Bedeutungszuwachs von Gesamtbetriebsräten gegenüber örtlichen Betriebsräten tatsächlich vorrangig als Diskrepanz zwischen Recht und gelebter Praxis begreifen lässt, ist alles andere als eindeutig und lässt sich auch mit den in der Folge präsentierten Daten nicht abschlieBend klären.

Mehr noch als die Frage nach der Effektivität, also der rechtskonformen Anwendung des Betriebsverfassungsgesetzes, analysiert dieser Beitrag die Implikationen, die die verbreitete Verlagerung von Regelungskompetenzen von der lokalen auf die Gesamtbetriebsratsebene für die Balance des dualen Systems der Arbeitnehmervertretung und damit für deren Qualität gerade auch auf der betrieblichen Ebene hat.

In diesem Punkt begründen Betriebsverfassung und Tarifautonomie als aufeinander bezogene Rechtssphären eine klare
Systematik und in der langjährigen Rechtspraxis ein hohes $\mathrm{Ma}$ an Regelungsdichte und Komplementarität (Schmidt/Trinczek 1991, 1999).

Betriebsräte sind für die unmittelbare Vertretung konkreter, situativer Beschäftigteninteressen im betrieblichen Alltag zuständig. Sie können sich dabei auf gesetzliche Mitwirkungs- und Mitbestimmungsrechte stützen, die im Grundsatz - wenn auch nicht immer in der Umsetzung - unabhängig von gewerkschaftlicher Vertretungsstärke und kollektiver Konfliktfähigkeit vor Ort geltend gemacht werden können und von Rechts wegen auch müssen. Gleichzeitig hängt die Handlungsfähigkeit von Betriebsräten entscheidend von der Rechtsetzungsmacht von Gewerkschaften ab, nicht nur weil diesen schon allein von

Martin Behrens, Dr. (USA), Wissenschaftler im WSI in der Hans-Böckler-Stiftung. Arbeitsschwerpunkte: Komparative industrielle Beziehungen, Arbeitsbeziehungen in den USA, Arbeitgeberverbände, Tarifpolitik. e-mail: martin-behrens@boeckler.de Jürgen Kädtler, PD Dr., Direktor am Soziologischen Forschungsinstitut Göttingen (SOFI) an der Georg-August-Universität. Arbeitsschwerpunkte: Industrielle Beziehungen, Tarifpolitik; Globalisierung, Soziologie der Finanzmärkte. e-mail: juergen.kaedtler@sofi.unigoettingen.de

Die Autoren danken Holger Alda, Wolfram Brehmer, Stefanie Kremer, Manfred Weiss, Christine Zumbeck und Nadine Zeibig für Anregungen und Anmerkungen zu diesem Beitrag. 
Gesetzes wegen die ausschließliche Zuständigkeit im Bereich der Tarifpolitik zukommt (BetrVG $\$ 77$ III), sondern auch, weil Gewerkschaften über die Kompetenz verfügen, die Bildung von Betriebsräten einzuleiten (BetrVG \$17 III).

Dies und der de facto hohe, mit der Betriebsgröße steigende Organisationsgrad von Betriebsräten resultieren in einer ausgesprochen effektiven Verschränkung der beiden Vertretungsarenen und Rechtssphären, mit den lokalen Betriebsräten als dem entscheidenden Scharnier. Als unmittelbare Adressaten der Beschäftigten vor Ort bilden sie die vorrangige Schnittstelle für die Aufnahme von Beschäftigteninteressen und für deren Konsolidierung und Transformation in gewerkschaftliche Vertretungsziele und Tarifforderungen. Und angesichts ihrer exklusiven Zuständigkeit für die situative Beschäftigtenvertretung und damit auch für die konkrete Umsetzung kollektiven Arbeitsrechts im Betrieb hängt es maßgeblich von ihnen ab, was daraus im Arbeitsalltag schließlich wird. Von daher überrascht es nicht, wenn Betriebsräten das Gros gewerkschaftlicher Mitgliederrekrutierung zugeschrieben wird (Behrens 2005a; Schmidt/Trinczek 1999).

\subsection{GESAMTBETRIEBSRÄTE UND BETRIEBSRÄTE}

Inwieweit die Bedeutungszunahme von Gesamtbetriebsräten und damit die Regulierungsfähigkeit des dualen Systems der Arbeitnehmervertretung beeinträchtigt wird, hängt davon ab, ob die Handlungsfähigkeit lokaler Betriebsräte vor Ort dadurch erhöht oder vermindert wird. Fördern Gesamtbetriebsräte die Arbeitsmöglichkeiten der lokalen Betriebsräte, indem sie ihre Handlungsvoraussetzungen verbessern? Oder werden der Handlungsspielraum und die Fähigkeit der lokalen Betriebsräte, den Belangen ihrer unmittelbaren Klientel angemessen Rechnung zu tragen, vermindert, mit der Konsequenz, dass sie weniger als deren Vertreter wahrgenommen werden, denn vielmehr als Agenturen der Gesamtbetriebsräte vor Ort?

Damit sind Effektivitäts- wie auch Legitimitätsfragen aufgeworfen. Ginge die Aufwertung von Gesamtbetriebsräten zulasten der örtlichen Betriebsräte mit einer weniger effektiven Vertretung der Belegschaftsinteressen einher, dann hätte dies Ausstrahlungseffekte auf das gesamte Arbeitsrecht, denn schließlich kommt den Be- triebsräten im Zuge des ,internal monitoring" eine herausragende Stellung bei der Kontrolle der Einhaltung von arbeitsrechtlichen Vorschriften z. B. im Bereich des Arbeits- und Gesundheitsschutzes zu (Weiss 2006).

Darüber hinaus könnte eine Delegation von Kompetenzen in Richtung Gesamtbetriebsrat wegen der längeren Repräsentationskette zu einer Entfremdung der kollektiven Vertretung von den Belegschaftsinteressen führen. So hat beispielsweise Wassermann darauf hingewiesen, dass es nicht ausreicht, die kollektive Handlungsmacht dort anzusiedeln „wo die Musik spielt“. Interessenvertretung schöpft ihre reale Stärke immer auch aus ihrer sozialen Nähe zu den Beschäftigten, also da zu sein, „wo die Leute sind“ (Wassermann 2002, S. 146). ${ }^{1}$ Letztlich können sich also Effektivitätsdefizite sowohl direkt aus der Verfehlung der Schutzfunktion von Rechtsnormen ergeben, aber auch Resultat von Entfremdungsprozessen sein, welche aus verfehlten Ordnungsvorschriften resultieren.

Wir werden uns daher in der Folge auf die Analyseebene der kollektiven Vertretung konzentrieren und dabei Fragen der Entfremdung des Betriebsrates in Form der institutionellen Einbettung des GBR in das System der industriellen Beziehungen thematisieren. Von besonderer Bedeutung erscheinen uns hierbei das Verhältnis der unterschiedlichen Betriebsratsebenen zueinander, aber auch ihr Verhältnis zu den Gewerkschaften sowie Kommunikationskanäle der Betriebsratsebenen mit der jeweiligen Belegschaft.

Wir gehen dabei in fünf Schritten vor. Abschnitt 2 stellt den der Analyse zugrunde liegenden Datensatz vor und gibt Auskunft über die Erhebungsmethoden. Abschnitt 3 überprüft, basierend auf den Ergebnissen der WSI-Betriebsrätebefragung zur Restrukturierung (2006), zunächst die Frage nach der Verteilung der Zuständigkeiten zwischen örtlichem Betriebsrat und Gesamtbetriebsrat. Der folgende Abschnitt 4 greift auf die Ergebnisse der WSI-Gesamtbetriebsrätebefragung zur Restrukturierung (2006) zurück und analysiert zentrale Strukturmerkmale der Gesamtbetriebsräte. Die aufgeworfene Entfremdungsthese wird in Abschnitt 5 im Rahmen einer multivariaten Analyse empirisch überprüft. In Abschnitt 6 werden die Ergebnisse diskutiert.

\section{Methode und Datengrundlage}

Die Analyse basiert auf der telefonischen Befragung (CATI) von 2.000 Betriebsräten und 283 korrespondierenden Gesamtbetriebsräten zum Thema der betrieblichen Restrukturierung, die vom Institut für angewandte Sozialwissenschaften (infas) im Auftrag des WSI durchgeführt wurde. Die Interviews wurden im Frühjahr 2006 zumeist mit den jeweiligen Betriebsratsvorsitzenden bzw. Gesamtbetriebsratsvorsitzenden geführt. Die Grundauswahl der Interviews erfolgte auf der Basis einer disproportionalen Zufallsstichprobe von 23.027 Adressen aus der Betriebsdatei der Bundesagentur für Arbeit (BA). Für die Befragung wurden nur Betriebsstätten aus der privaten Wirtschaft berücksichtigt, die 20 oder mehr Beschäftigte aufweisen. Die Ausschöpfung der Stichprobe im Bereich der örtlichen Betriebsräte betrug nach Abzug der neutralen Ausfälle (z. B. kein Betriebsrat, fehlende Telefonnummer) 49,6\%.

Die Stichprobe für die Befragung der Gesamtbetriebsräte leitet sich aus der Betriebsratserhebung ab. Mit dem ersten Kontakt (Rekrutierung) wurden die örtlichen Betriebsräte gefragt, ob ein für sie zuständiger Gesamtbetriebsrat besteht. War dies der Fall, wurden die befragten Zielpersonen um die Übermittlung der Angaben $\mathrm{zu}$ diesem GBR-Gremium gebeten und diese zusammen mit den Kontaktdaten des zuständigen GBR-Vorsitzenden in einer gesonderten Datenbank erfasst. Für die Steuerung der Interviews wurden zwei Grundprinzipien angewendet. Zunächst

\footnotetext{
Dieser Spagat und die daraus resultierenden Interessenkonflikte wurden im Kontext des jüngsten VW-Skandals schmerzlich in Erinnerung gerufen. So berief sich der ehemalige Gesamt- und Konzernbetriebsratsvorsitzende Volkert in seiner Selbstrechtfertigung vor Gericht auf die Parität seiner Position und Person mit den handelnden Managementvertretern im Konzern. Wer dauerhaft mit diesen verhandle und schwierige Entscheidungen treffe, so Volkert im Verfahren vor dem Landgericht Braunschweig, müsse sich auch in Hinsicht auf Vergütung und Privilegien diesem Referenzrahmen zuordnen dürfen.Offensichtlich ging in diesem Extremfall die Entfremdung bereits so weit, dass sie zu Machtmissbrauch und einer vollständigen Durchtrennung der Delegationskette geführt hat.
} 
Tabelle 1: Zuständigkeiten bei Aushandlung von Betriebsvereinbarungen

\begin{tabular}{|c|c|c|c|c|c|}
\hline Gegenstandsbereich & $\begin{array}{c}\text { Nur örtlicher } \\
\text { BR }\end{array}$ & GBR & $\begin{array}{l}\text { Andere } \\
\text { Ebene }\end{array}$ & Gemeinsam & Fallzahl \\
\hline Einstellungen & 43 & 41 & 4 & 13 & 329 \\
\hline Entlassungen & 32 & 46 & 5 & 18 & 288 \\
\hline Versetzungen/Umsetzungen & 35 & 43 & 3 & 19 & 348 \\
\hline Eingruppierung & 45 & 40 & 4 & 12 & 356 \\
\hline Entlohnung & 38 & 43 & 5 & 14 & 410 \\
\hline Zielvereinbarungen & 29 & 50 & 5 & 15 & 338 \\
\hline Arbeitszeitverkürzung & 49 & 32 & 1 & 17 & 270 \\
\hline Arbeitszeitverlängerung & 49 & 36 & 2 & 13 & 303 \\
\hline Arbeitszeitkonten & 54 & 30 & 2 & 14 & 573 \\
\hline Mehrarbeit & 59 & 30 & 2 & 9 & 470 \\
\hline Ausbildung & 30 & 54 & 4 & 13 & 335 \\
\hline Weiterbildung & 30 & 50 & 5 & 15 & 405 \\
\hline Familienfreundlichkeit & 24 & 55 & 7 & 15 & 164 \\
\hline Frauenförderung & 15 & 64 & 6 & 16 & 175 \\
\hline Technikgestaltung & 18 & 57 & 6 & 18 & 288 \\
\hline Arbeitsorganisation & 44 & 39 & 2 & 15 & 265 \\
\hline Arbeitsschutz & 27 & 48 & 7 & 17 & 439 \\
\hline Beschäftigungssicherung & 27 & 47 & 6 & 21 & 349 \\
\hline Sucht & 34 & 44 & 6 & 15 & 350 \\
\hline Datenschutz & 14 & 59 & 8 & 20 & 589 \\
\hline Betriebliche Sozialleistungen & 21 & 56 & 5 & 18 & 534 \\
\hline Vorschlagswesen & 26 & 52 & 6 & 17 & 387 \\
\hline Sonstige & 43 & 41 & 4 & 12 & 362 \\
\hline
\end{tabular}

einmal wurde darauf geachtet, dass die Interviews für die Gremien „örtlicher Betriebsrat" und „Gesamtbetriebsrat" mit jeweils unterschiedlichen Personen geführt wurden. Dies erschien notwendig, um die aus Ämterhäufung resultierenden Rollenkonflikte und sich daraus ergebene Verzerrungen im Antwortverhalten zu vermeiden. Sollte die kontaktierte Person für das Interview mit dem örtlichen Betriebsrat gleichzeitig auch den Vorsitz im GBR-Gremium innehaben, wurde das Interview mit dem örtlichen BR mit einem anderen Mitglied durchgeführt. Als zweites Grundprinzip der Steuerung wurde darauf geachtet, GBR-Interviews nur dann durchzuführen, wenn bereits ein Interview mit dem zuständigen örtlichen Betriebsrat vorlag. Durch dieses Matching wurde eine direkte Zuordnung der jeweiligen Antworten auf den unterschiedlichen Betriebsratsebenen ermöglicht. Insgesamt konnten in der Kontaktierungsphase 751 unterschiedliche Gesamtbetriebsratsgremien identifiziert werden. Von diesen Adressen wurden insgesamt 334 eingesetzt, also ausschließlich solche Gremien, für die bereits ein korrespondierendes Interview mit dem örtlichen BR vorlag. Die Teilnahmebereitschaft der Gesamtbetriebsräte erwies sich mit 85,6\% als sehr hoch. und zwingend zwischen den Betriebsparteien und sind somit die wohl sichtbarsten Produkte des Interessenvertretungshandelns. Das Bestehen von Betriebsvereinbarungen zu bestimmten Themen wurde auf der Ebene des örtlichen Betriebsrates erhoben. ${ }^{2}$ Die inhaltlichen Themenfelder und Kategorien wurden auf Basis früherer WSIBetriebsrätebefragungen entwickelt und im Rahmen eines umfangreichen Pre-Tests weiter angepasst. Letztlich wurden 23 unterschiedliche Themenbereiche inklusive einer offenen Kategorie abgefragt. In den
Fällen, in denen die befragten örtlichen Betriebsräte zuvor angegeben hatten, dass für ihren Bereich eine weitere Vertretungsebene besteht (GBR, Konzernbetriebsrat (KBR) oder Europäischer Betriebsrat (EBR)), wurde darüber hinaus um Auskunft darüber gebeten, auf welcher Betriebsratsebene die Vereinbarung überwiegend abgeschlossen wurde. Die zur Auswahl stehenden Kategorien waren „örtlicher Betriebsrat" „Gesamtbetriebsrat", „,andere Ebene“ oder "gemeinsam durch mehrere Ebenen“. Tabelle 1 dokumentiert die Verteilung der Antworten.

Insgesamt fällt zunächst auf, dass die Ebenen der KBR und EBR, welche in der Kategorie „andere Ebene“ zusammengefasst wurden, eine nur untergeordnete Rolle spielen. Weiterhin gaben die Betriebsräte in den einzelnen Themenfeldern für 9 bis $21 \%$ der Fälle an, dass Vereinbarungen gemeinsam durch mehrere Ebenen abgeschlossen wurden. Wir interpretieren diese Fälle als Ausdruck für die Breite der von uns angebotenen Antwortkategorien. So ist es durchaus möglich, dass es beispielsweise zum Thema Sucht im Unternehmen mehrere Betriebsvereinbarungen gibt, von denen einige vom örtlichen Betriebsrat und andere vom Gesamtbetriebsrat ausgehandelt und abgeschlossen wurden. Vergleicht man nun die beiden verbleibenden Kategorien „örtlicher Betriebsrat" und „Gesamtbetriebsrat", so ergeben sich zum Teil erstaunliche Ergebnisse: Der Anteil der Primärzuständigkeit des Gesamtbetriebsrates ist über alle Themenfelder hinweg sehr hoch und unterschreitet in keinem einzigen Themenfeld den Anteil von $30 \%$. In insgesamt 9 von 23 Themenfeldern geben mehr als $50 \%$ der antwortenden örtlichen Betriebsräte an, dass Betriebsvereinbarungen durch den GBR abgeschlossen wurden. Hingegen kam in nur 2 Feldern in mehr als $50 \%$ der Fälle dem örtlichen Betriebsrat eine Primärzuständigkeit zu. Weiterhin wurden in nur 8 von 23 Themenfel-

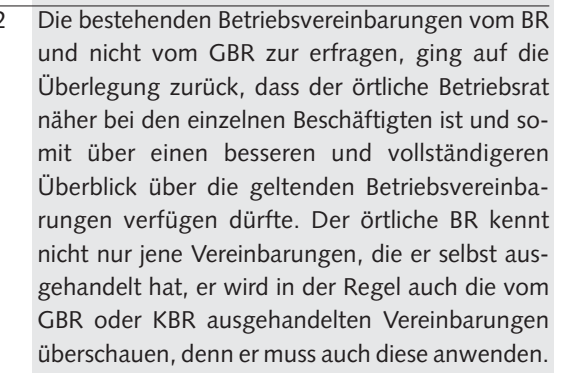

2 Die bestehenden Betriebsvereinbarungen vom BR und nicht vom GBR zur erfragen, ging auf die Überlegung zurück, dass der örtliche Betriebsrat näher bei den einzelnen Beschäftigten ist und somit über einen besseren und vollständigeren Überblick über die geltenden Betriebsvereinbarungen verfügen dürfte. Der örtliche BR kennt nicht nur jene Vereinbarungen, die er selbst ausgehandelt hat, er wird in der Regel auch die vom GBR oder KBR ausgehandelten Vereinbarungen überschauen, denn er muss auch diese anwenden. 
dern die dort ausgehandelten Betriebsvereinbarungen mehrheitlich durch den örtlichen Betriebsrat unterschrieben, in den verbleibenden 15 Feldern hat der GBR die Kompetenz in der Mehrzahl der Fälle an sich gebunden. Als Refugien der mehrheitlichen Zuständigkeit der örtlichen Betriebsräte erweisen sich insbesondere alle Fragen, welche mit Arbeitszeit zusammenhängen (Arbeitszeitverkürzung und -verlängerung, Arbeitszeitkonten, Mehrarbeit), aber auch Eingruppierung und Arbeitsorganisation.

Insgesamt erweisen sich die Befunde als recht eindeutig. Überall dort, wo es kein explizites Erfordernis zur Anpassung der Regelungsmaterien an die betrieblichen Bedingungen gibt, wird die Regelungskompetenz mehrheitlich durch den GBR wahrgenommen. Die eigentliche Intention von Gesetz und Rechtsprechung, den GBR nur als subsidiäre Instanz ins Spiel zu bringen, die den örtlichen Betriebsräten „nicht übergeordnet" ist ( $\$ 50$ I BetrVG), findet sich in der von uns beobachteten Praxis nur eingeschränkt wieder.

Diese Befunde decken sich in wesentlichen Teilen mit jenen einer Studie von Rancke (1982), der in einer schriftlichen Befragung Gesamtbetriebsräte von Großunternehmen zur Verteilung der Zuständigkeiten zwischen örtlichen Betriebsräten, Gesamt- und Konzernbetriebsräten befragt hatte. So kam in 13 von 18 der von Rancke abgefragten Themenfelder die Regelungszuständigkeit mehrheitlich den Gesamtbetriebsräten $\mathrm{zu}$, während der örtliche Betriebsrat in Fragen der Arbeitszeitregelung, Urlaubsplanung, Arbeitsordnung und Leistungsvergütung das Heft in der Hand hielt (Rancke 1982, S. 460).

\section{Strukturmerkmale der Gesamtbetriebsräte}

Für die Bewertung von Fragen der Entfremdung und Verpflichtungsfähigkeit erscheint es zunächst notwenig, die Organisations- und Verflechtungsstrukturen der Gesamtbetriebsräte mit den örtlichen Betriebsräten und Belegschaften näher zu analysieren. Gemäß $\$ 47$ I BetrVG ist ein Gesamtbetriebsrat zu bilden, wenn in einem Unternehmen mehrere Betriebsräte bestehen. Insgesamt verweisen 38,4 \% der von uns befragten örtlichen Betriebsräte auf einen für sie zuständigen Gesamtbetriebsrat. Dies ist exakt der Prozentsatz, den wir bereits in der vorangegangenen Befragung im Jahr 2004/05 ermitteln konnten (Behrens 2005b, S. 639). Vergleicht man nun die Komplexität der jeweiligen GBRGremien, d.h. die Frage, wie viele örtliche Betriebsräte durch den jeweils zuständigen Gesamtbetriebsrat repräsentiert werden, ergeben sich beachtliche Differenzen. Wie Abbildung 1 belegt, repräsentieren über $61 \%$ der von uns befragten Gesamtbetriebsräte zwischen zwei und zehn örtliche Betriebsräte während weitere $30 \%$ der GBR bis zu 50 örtliche Betriebsräte zusammenfassen. Immerhin noch mehr als $8 \%$ der befragten GBR vertreten 51 und mehr örtliche Betriebsräte, wobei in der Spitze bis zu 300 örtliche Betriebsräte durch einen GBR erfasst werden.

Nun ergibt sich zwar aus dem Gesetz eine Verpflichtung zur Wahl eines GBR, wenn innerhalb eines Unternehmens mehr als zwei örtliche Betriebsräte gebildet wurden. Einen Zwang zur Wahl eines örtlichen Betriebsrates selbst gibt es allerdings nicht. Es stellt sich daher auf Unternehmensebene die Frage nach den sogenannten weißen Flecken der Interessenvertretung. Um diese quantifizieren zu können, haben wir die GBR auch danach gefragt, ob es in ihrem Unternehmen Betriebsstätten gibt, für die keine örtlichen Betriebsräte gebildet wurden. Immerhin 40,6\% der befragten GBRVorsitzenden bejahten dies. Eine weitere Aufschlüsselung ergab, dass im Bereich der GBR mit mindestens einer Betriebsstätte ohne örtlichen BR im Mittel für $27 \%$ dieser Betriebsstätten keine örtliche Interessenvertretung gebildet wurde. Die Anteile der „weißen Flecken“ an der Interessenvertretungsdomäne der jeweiligen GBR reichen hierbei von $1 \%$ bis $85 \%$. Dieser Befund verweist darauf, dass betriebsratsfreie Bereiche nicht allein in solchen Unternehmen und deren Betrieben entstehen, in denen eine formale Vertretung der Belegschaftsinteressen ganz fehlt, sondern in beachtlichem Maße auch Resultat der unvollständigen Erschließung sein können. $\mathrm{Ob}$ nun die weißen Flecken in teilerschlossenen Unternehmen Resultat solcher Vermeidungsstrategien sind, wie Beispiele aus dem Einzelhandel nahelegen, oder aber andere Einflussfaktoren am Werke sind, muss hier mangels weitergehender Informationen offen bleiben.

Wie die eben präsentierten Befunde bereits andeuten, kann man im Bereich der

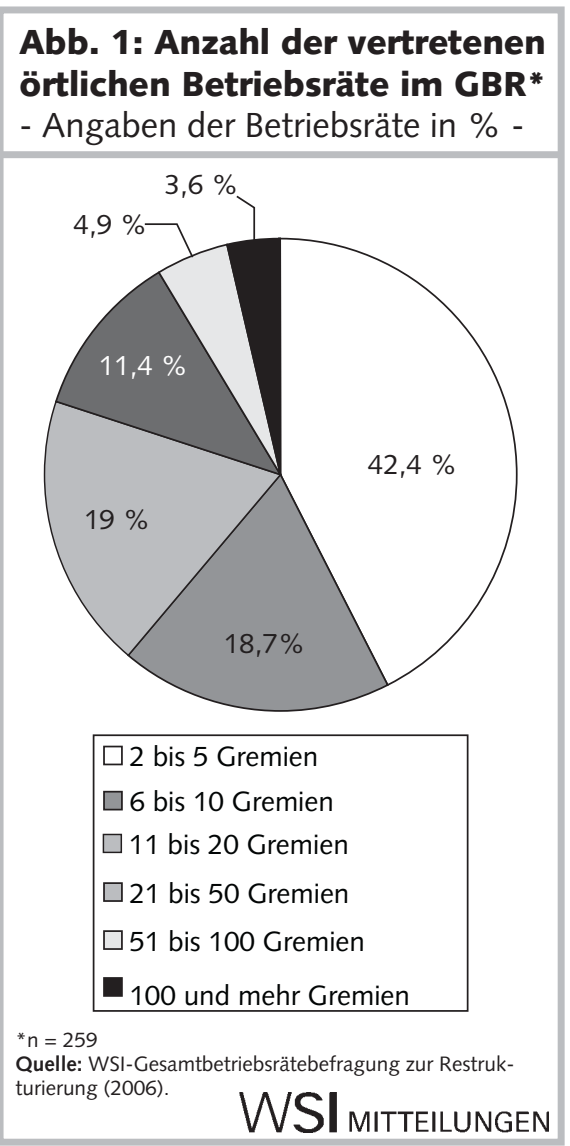

Gesamtbetriebsräte, anders noch als bei den örtlichen Betriebsräten, nicht von einer einheitlichen Einbettung in das System der industriellen Beziehungen ausgehen. Dies trifft nicht nur für die Frage zu, ob in den Betriebsstätten des Unternehmens überhaupt ein örtlicher Betriebsrat gebildet wurde, sondern auch für die Tarifbindung und die zuständigen Gewerkschaften. So gaben immerhin $15 \%$ der befragten $\mathrm{Ge}-$ samtbetriebsräte an, dass mehrere Gewerkschaften „überwiegend“ die Beschäftigten im Unternehmen organisieren. Darüber hinaus haben wir die Gesamtbetriebsräte gefragt, welche Formen der Tarifbindung im Unternehmen existieren (Antwortkategorien: Flächentarifvertrag, Haustarif, Ergänzungstarif, keine Tarifbindung) und auf wie viele Betriebsstätten die jeweiligen Formen Anwendung finden. Die Auswertung zeigte, dass in immerhin einem Viertel der untersuchten Unternehmen multiple Formen der Tarifbindung vorliegen, wobei es eine Vielzahl unterschiedlicher Kombinationen gibt. 


\section{Führt die Verlagerung von Kompetenzen zur Entfremdung?}

Ein GBR kann sich in vielfacher Form als entfremdet erweisen: Drei Ebenen sind hierbei relevant. Erstens, die Entfremdung des GBR von den Belegschaften, d.h. den Beschäftigten in den Betrieben. Entfremdung bedeutet hierbei eine Verselbstständigung gegenüber den Beschäftigten und ihren Interessen, die sich letztlich auch in einer größeren kommunikativen Distanz ausdrückt. Zweitens können sich aber auch Entfremdungsprozesse ergeben, die aus dem Delegationsprinzip selbst resultieren, d.h. zwischen dem örtlichen Betriebsrat und dem GBR auftreten. Drittens sind aber auch Entfremdungsprozesse von Bedeutung, die erst durch die Dualität des deutschen Systems der Arbeitsbeziehungen an Bedeutung gewinnen (Abschnitt 1.1), d.h. die das Verhältnis zwischen Betriebsräten und der zuständigen Gewerkschaft berühren. Zur Bestimmung der Einflussfaktoren für unterschiedliche Formen der Entfremdung präsentieren wir multivariate Schätzungen.

Fragen der Distanz zwischen GBR und den Belegschaften werden in Form der direkten Kontaktaufnahme zwischen beiden operationalisiert, wobei hier insbesondere die Präsenz von Betriebsräten an den Arbeitsplätzen der Beschäftigten von Bedeutung ist. Aspekte der Autonomisierung der Betriebsratsebenen von den Gewerkschaften werden durch die Bewertung dieses Verhältnisses durch die befragten Betriebsräte berücksichtigt. Wie sich bereits in den vorangegangenen Abschnitten andeutete, lassen sich einige Grundannahmen über die Arbeit des örtlichen Betriebsrates, wie z. B. eine einheitliche Tarifbindung innerhalb des jeweiligen Organisationsbereichs, nicht umstandslos auf die Arbeit der Gesamtbetriebsräte übertragen. Die folgenden Analyseschritte werden daher jeweils für den örtlichen Betriebsrat sowie den Gesamtbetriebsrat getrennt vorgenommen.

\subsection{ENTFREMDUNG DER BETRIEBS- RÄTE VON DEN BELEGSCHAFTEN}

In einem ersten Analyseschritt wird zunächst der Frage nach den Bestimmungsfaktoren für die Kontaktaufnahme des
GBR zur Belegschaft nachgegangen. In beiden Teilbefragungen wurden die örtlichen Betriebsräte und Gesamtbetriebsräte danach gefragt, welche direkten oder indirekten Kontaktmöglichkeiten zur Belegschaft sie nutzen. ${ }^{3}$ Der Durchführung von Betriebsbegehungen, die als abhängige Variable in den beiden folgenden Schätzungen verwandt wurde, kommt hierbei besondere Bedeutung zu, weil sie den Kontakt zwischen Beschäftigten und Interessenvertretern im Kontext der jeweiligen Arbeitssituation herstellt. Insgesamt $71 \%$ der örtlichen Betriebsräte gaben an, regelmäßig solche Begehungen durchzuführen, während dieser Anteil bei den Gesamtbetriebsräten immerhin noch $65 \%$ betrug.

Als unabhängige Variable wurde zunächst ein Indikator für die Kompetenzverteilung zwischen örtlichem Betriebsrat und Gesamtbetriebsrat in die Schätzung einbezogen. So bringt die Variable „Zuständigkeit" die Verteilung der Zuständigkeiten zwischen beiden Betriebsratsebenen zum Ausdruck. Hierzu wurde wiederum auf die in Tabelle 1 dargestellte Verteilung der Zuständigkeiten zur Aushandlung von Betriebsvereinbarungen zurückgegriffen. Fälle in denen eine Betriebsvereinbarung vom örtlichen Betriebsrat ausgehandelt wurde, wurden mit 1 , solche bei denen der Gesamtbetriebsrat oder eine „höhere“ Betriebsratsebene (KBR, EBR) Verhandlungspartner waren mit -1 kodiert. Fälle bei denen eine bestimmte Form der Betriebsvereinbarung nicht vorliegt oder zwar vorliegt, jedoch gemeinsam durch mehrere Ebenen abgeschlossen wurde, wurden auf 0 gesetzt. Durch Aufaddieren entsteht eine Zuständigkeitsvariable die, bezogen auf den einzelnen örtlichen Betriebsrat, den Wertebereich -23 bis 21 (Mittelwert $-2,4$ ) annimmt, wobei negative Werte ein quantitatives Übergewicht der Zuständigkeit von GBR, KBR und EBR zum Ausdruck bringen. Diese Variable ist ein Indikator für die Kompetenzverteilung zwischen den Betriebsratsebenen, wobei „Kompetenzverteilung " nicht notwendigerweise ausdrückt, dass eine solche Aufteilung der Zuständigkeiten gegen geltende betriebsverfassungsrechtliche Vorschriften erfolgte. ${ }^{4}$

Weitere Kontrollvariablen berücksichtigen den dichotomisierten Organisationsgrad des Betriebsrats- bzw. Gesamtbetriebsratsgremiums (1 wenn dieser größer als $70 \%$ ist) sowie die durch den Betriebsrat selbst eingeschätzte Distanz zu den Gewerkschaften, eine Variable die den Wert 1 annimmt, wenn die Befragten der folgenden Aussage zustimmen: „Der Betriebsrat/Gesamtbetriebsrat ist die Vertretung der Gewerkschaft im Betrieb/Unternehmen ". ${ }^{5}$ Es erscheint sinnvoll, beide Aspekte in die Schätzung einzubeziehen, denn die eigene Gewerkschaftsmitgliedschaft und die Identifizierung mit der gewerkschaftlichen Organisation sind keineswegs identische Kategorien.

Die ökonomische Situation des Betriebs bzw. Unternehmens ( 1 bei schlechter Auftragslage) wird berücksichtigt, ebenso wie mögliche Konflikte mit dem Arbeitgeber. Die Konfliktvariable nimmt dabei den Wert 1 an, wenn die befragten Betriebsräte angeben, der Arbeitgeber würde sie häufig bei der Ausübung ihrer Mitbestimmungsrechte behindern.

Weiterhin enthalten beide Schätzmodelle Branchendummies sowie die logarithmierte Beschäftigtenzahl im Betrieb bzw. Unternehmen. In den statistischen Modellen für den GBR wird darüber hinaus die Anzahl der durch den GBR erfassten örtlichen Betriebsratsgremien in die Schätzung einbezogen. Dies erscheint sinnvoll, denn allein die Zahl der Beschäftigten im Unternehmen sagt noch nichts darüber aus, auf wie viele Betriebe diese Belegschaft verteilt ist, d.h. in welcher Form die „Betriebsgröße" hier überhaupt durch die handelnden Akteure erfahrbar wird.

3 Vorgegeben wurden: Schriftliche Betriebsratsinfos, Aushänge, Betriebsversammlungen (Auftritte des Gesamtbetriebsrates auf Betriebsversammlungen), Betriebsbegehungen, Sprechstunden, Teilnahme von Beschäftigten an (Gesamt-)Betriebsrätearbeitskreisen, Zusammenarbeit mit gewerkschaftlichen Vertrauensleuten, Beiträge des (Gesamt-) Betriebsrates in der Betriebszeitung, und E-Mail/betriebsinternes Netz.

4 Für alle in der Folge präsentierten Schätzungen wurden alternative Regressionen durchgeführt, die statt der hier eingeführten Zuständigkeitsvariablen eine zensierte Variable einbezogen haben, welche lediglich jene 6 Themenfelder berücksichtigt, für die eine Primärzuständigkeit des örtlichen Betriebsrates anzunehmen ist (Einstellungen, Entlassungen, Versetzungen/Umsetzungen, Arbeitszeitverkürzung und -verlängerung, Arbeitsschutz). Mit Ausnahme geringer Unterschiede der Koeffizienten reproduzierten die Alternativschätzungen die hier präsentierten Ergebnisse.

5 Insgesamt wurde das Interessenvertretungsselbstverständnis auf einer 6er Skala erhoben, wobei der Wert 1 besonders starke Zustimmung und der Wert 6 besonders starke Ablehnung zum Ausdruck bringt. In den folgenden Analysen wurden die Werte 1 und 2 zusammengefasst. 
Die Modelle im Bereich der Gesamtbetriebsräte beziehen alle Fälle in die Schätzung ein, die Modelle im Bereich der örtlichen Betriebsräte nur solche, in denen die befragten Interessenvertreter überhaupt einen zuständigen GBR benannt haben. Die Ergebnisse der Schätzungen werden in der Tabelle 2 dargestellt.

Die Schätzung für den Organisationsbereich des örtlichen Betriebsrates weist zunächst einen positiven, aber nicht signifikanten Zusammenhang zwischen der Zuständigkeitsverteilung und der regelmäßigen Durchführung von Betriebsbegehungen auf. Signifikante Assoziationen ergeben sich bezüglich des gewerkschaftlichen Organisationsgrades des Betriebsratsgremiums (Irrtumswahrscheinlichkeit 0,1\%) und der Beschäftigtenzahl (0,1\%). Wenn das Betriebsratsgremium überdurchschnittlich gut organisiert ist (70\% und mehr), steigt - bei statistischer Kontrolle aller anderen in die Schätzung einbezogenen Variablen - die Wahrscheinlichkeit, dass regelmäßige Betriebsbegehungen durchgeführt werden. Auch steigt die Wahrscheinlichkeit einer solchen Kontaktaufnahme mit der Betriebsgröße an. Ob nun solche Begehungen von den Beschäftigten genutzt werden, um Probleme zu besprechen oder Wünsche an die Interessenvertreter heranzutragen, oder nur ein symbolträchtiges „Bad in der Menge" darstellen, welches lediglich die Nähe der Betriebsratsmitglieder zur Belegschaft vorgaukelt, ist damit noch nicht ausgemacht. Ins Auge fällt weiterhin ein deutlich negativer Einfluss des Branchendummies für den Bereich Kredit/Versicherungen. Im Vergleich zur Referenzkategorie (sonstige Dienstleistungen) ist hier die Wahrscheinlichkeit von Betriebsbegehungen unterdurchschnittlich (Signifikanz auf dem $1 \%$-Niveau).

Ein Vergleich zu den Schätzergebnissen für den Bereich der Gesamtbetriebräte (rechte Spalte von Tabelle 2) ergibt einige bemerkenswerte Unterschiede. So weist nun die Zuständigkeitsverteilung eine negative und signifikante (5\%-Niveau) Assoziation mit der Wahrscheinlichkeit von Betriebsbegehungen auf. Sie sinkt, wenn der örtliche Betriebsrat mehrheitlich die Kompetenzen zur Aushandlung von Betriebsvereinbarungen an sich gebunden hat und sie steigt, wenn eine höhere Betriebsratsebene (GBR, KBR, EBR) die Zuständigkeit für sich reklamiert. Möglicherweise handelt es sich bei dem aufgefundenen $\mathrm{Zu}$ sammenhang um einen Kompensationsef-

Tabelle 2: Kontakte1) von BR und GBR zur Belegschaft

\begin{tabular}{|c|c|c|}
\hline & Örtlicher Betriebsrat & Gesamtbetriebsrat \\
\hline Zuständigkeit & 010 &,$- 041^{*}$ \\
\hline Organisationsgrad BR/GBR & $864 * * *$ & $674^{*}$ \\
\hline Schlechte Auftragslage & 138 &, 180 \\
\hline Behinderung BR & ,381 & ,387 \\
\hline Anzahl örtliche BR-Gremien & - &,$- 018 * *$ \\
\hline Beschäftigtenzahl (log.) &, $765^{* * *}$ &,- 013 \\
\hline Distanz zur Gewerkschaft & ,299 &, 434 \\
\hline \multicolumn{3}{|c|}{ Sonstige Dienstleistungen. (Referenz) } \\
\hline Grundstoffe & 1,229 & - \\
\hline Investitionsgüter &,- 287 & - \\
\hline Verbrauchsgüter &,- 109 & - \\
\hline Bau &,- 492 & - \\
\hline Handel &,- 049 & - \\
\hline Verkehr/Nachrichten & ,245 & - \\
\hline Kredit/Versicherungen & $-1,009 * *$ & - \\
\hline Sonstige Branchen & ,427 & - \\
\hline Landw./Bergbau & - &, 428 \\
\hline Produzierendes Gewerbe & - & ,823* \\
\hline Bau & - &, 552 \\
\hline Handel & - & $2,737 * * *$ \\
\hline Verkehr/Kredit & - & ,748 \\
\hline Konstante & $-2,377 * * *$ &,- 464 \\
\hline $\begin{array}{l}\text { Nagelkerke R2 } \\
\text { gültige Fälle }\end{array}$ & $\begin{array}{l}0,290 \\
772\end{array}$ & $\begin{array}{l}0,192 \\
274\end{array}$ \\
\hline \multicolumn{3}{|c|}{$\begin{array}{l}\text { */**/*** statistisch signifikant auf dem } 5 / 1 / 0,1 \% \text {-Niveau. } \\
\text { 1) abhängige Variable: Führt regelmäßig Betriebsbegehungen durch. }\end{array}$} \\
\hline $\begin{array}{l}\text { Quelle: WSI-Betriebsrätebefragung } \\
\text { WSI-Gesamtbetriebsrätebefragung }\end{array}$ & & MITTEILUNG \\
\hline
\end{tabular}

fekt, wonach sich der GBR veranlasst sieht, seine Politik der Belegschaft gegenüber zu erklären oder zumindest Präsenz zu zeigen, wenn er in einer Mehrzahl der Themenbereiche Kompetenzen wahrnimmt.

Die Schätzung ergibt weiterhin einen negativen Einfluss der Anzahl der örtlichen Betriebsratsgremien (1\% Signifikanz), d.h. mit steigender Anzahl der durch den GBR zusammengefassten örtlichen Betriebsräte sinkt die Wahrscheinlichkeit von Betriebsbegehungen. Die im Vergleich zur Referenzkategorie größere Wahrscheinlichkeit von Begehungen im Handel (signifikant auf dem $1 \%$-Niveau) erklärt sich möglicherweise aus einem Selektionseffekt. Da im filialisierten Einzelhandel Betriebsratsgründungen oft nur gegen massiven Widerstand des Managements erfolgen, könnte sich der GBR zur verstärkten Pflege der Kontakte mit den Belegschaften veranlasst sehen.

\subsection{SCHÄTZMODELLE ZUR ENTFREMDUNG DER BETRIEBSRÄTE VON DEN GEWERKSCHAFTEN}

Das Verhältnis zwischen Betriebsräten und Gewerkschaften wurde über die Variable „Distanz zur Gewerkschaft“ erhoben, welche auf einer Selbsteinschätzung der be- fragten Interessenvertretungen beruht. Das hier als abhängige Variable verwendete Merkmal basiert - wie in den vorangegangenen Schätzungen - auf den Einschätzungen zur Aussage: „Der Betriebsrat/Gesamtbetriebsrat ist die Vertretung der Gewerkschaft im Betrieb/Unternehmen“. Während immerhin 28,5\% der örtlichen Betriebsräte dieser Aussage zustimmten, waren dies bei den Gesamtbetriebsräten nur 19,4 \%. Diese Diskrepanz in den Einschätzungen ist umso erstaunlicher, als der durchschnittliche Organisationsgrad der Mitglieder der befragten Gesamtbetriebsräte (nur DGB-Gewerkschaften) mit 77,9 \% fast 10 Prozentpunkte über jenem der korrespondierenden örtlichen Betriebsräte liegt $(68,5 \%)$, d.h., dass Gesamtbetriebsräte trotz ihrer vergleichsweise größeren gewerkschaftlichen Verankerung qua Mitgliedschaft größeren Wert auf ihre Unabhängigkeit von den Gewerkschaften legen.

Neben den genannten Variablen zur Zuständigkeitsverteilung, wurden alle bereits bekannten Variablen aus den vorangegangenen Schätzungen zum Kontaktverhalten der Betriebsratsebenen aufgenommen. Zusätzlich kontrollieren beide Modelle die Anzahl der Kontaktmöglichkeiten, die GBR und örtlicher Betriebsrat nutzen sowie ihre Bewertung der gewerkschaftli- 
Tabelle 3: Distanz ${ }^{1)}$ von BR und GBR zur Gewerkschaft

\begin{tabular}{|c|c|c|}
\hline & Örtlicher Betriebsrat & Gesamtbetriebsrat \\
\hline Zuständigkeit & ,010 & ,059* \\
\hline Organisationsgrad Betriebsrat ( 1 wenn $>70 \%$ ) &, $550 * *$ &,- 060 \\
\hline Bewertung Gewerkschaftsunterstützung &, $315^{* * *}$ &, $519 * *$ \\
\hline Schlechte Auftragslage &,- 471 &,- 204 \\
\hline Behinderung Betriebsrat &,- 150 & $-1,247$ \\
\hline Anzahl örtliche Betriebsratsgremien & - & ,002 \\
\hline Beschäftigtenzahl (log.) &,$- 194 * *$ &,- 091 \\
\hline Anzahl Kontaktmöglichkeiten &, $199 * *$ &, $220^{*}$ \\
\hline \multicolumn{3}{|l|}{ Sonstige Dienstleistungen (Referenz) } \\
\hline Grundstoffe &, 445 & - \\
\hline Investitionsgüter & $1,029 * *$ & - \\
\hline Verbrauchsgüter &, 568 & - \\
\hline Bau & ,358 & - \\
\hline Handel &, $625^{*}$ & - \\
\hline Verkehr/Nachrichten &, 579 & - \\
\hline Kredit/Versicherungen &,- 189 & - \\
\hline Sonstige Branchen &,- 307 & - \\
\hline Landwirtschaft/Bergbau & - & 1,246 \\
\hline Produzierendes Gewerbe & - &, 650 \\
\hline Bau & - & ,291 \\
\hline Handel & - & ,826 \\
\hline Verkehr/Nachrichten/Kredit & - & 1,000 \\
\hline Konstante & $-2,835^{* * *}$ & $-4,513 * * *$ \\
\hline $\begin{array}{l}\text { Nagelkerke R² } \\
\text { gültige Fälle }\end{array}$ & $\begin{array}{l}0,163 \\
713\end{array}$ & $\begin{array}{l}0,159 \\
264\end{array}$ \\
\hline \multicolumn{3}{|c|}{$\begin{array}{l}* / * * / * * * \text { statistisch signifikant auf dem } 5 / 1 / 0,1 \% \text {-Niveau. } \\
\text { 1) abhängige Variable: Distanz zur Gewerkschaft (1 wenn Identifikation). }\end{array}$} \\
\hline $\begin{array}{l}\text { Quelle: WSI-Betriebsrätebefragung zur Restrukturierung ( } 2 \mathrm{C} \\
\text { WSI-Gesamtbetriebsrätebefragung zur Restrukturierung ( } 20\end{array}$ & & MITTEILUNGEN \\
\hline
\end{tabular}

chen Unterstützung (Antwortmöglichkeiten reichen von $1=$ mangelhaft bis $5=\mathrm{sehr}$ gut). Wir gehen davon aus, dass sich ein Betriebsrat eher mit der Gewerkschaft identifiziert, wenn er mit deren Unterstützungsleistungen zufrieden ist.

Wie schon im bereits präsentierten Kontaktmodell (Tabelle 2) ist bei den örtlichen Betriebsräten auch bei der Frage der Distanz zur Gewerkschaft der Einfluss der Zuständigkeitsverteilung zwischen den Betriebsratsebenen positiv, aber nicht signifikant, d.h. er kann nicht sinnvoll interpretiert werden (Tabelle 3). Weiterhin steigt die Bereitschaft, sich mit der Gewerkschaft zu identifizieren, wenn der gewerkschaftliche Organisationsgrad des örtlichen Betriebsrats hoch ist (über $70 \%$ ) (signifikant auf dem $1 \%$-Niveau) und die Unterstützung der Gewerkschaft positiv bewertet wird (0,1 \% Signifikanz). Die Anzahl der vom BR genutzten Kontaktmöglichkeiten zur Belegschaft weist eine positive Assoziation mit der Identifikation mit der Gewerkschaft auf (1\% Signifikanz), während die Wahrscheinlichkeit einer solchen Identifikation mit steigender Betriebsgröße abnimmt (signifikant auf dem $1 \%$-Niveau). Dies ist ein bemerkenswerter Befund der darauf verweist, dass örtliche Betriebsräte sich eine gute Bewertung der Gewerkschaftsunterstützung positiv auf die Wahrscheinlichkeit einer Identifikation mit der Gewerkschaft auswirkt (signifikant auf dem $1 \%$-Niveau), findet sich kein signifikanter Effekt des gewerkschaftlichen Organisationsgrades, der Koeffizient ist sogar negativ. Einen positiven Einfluss übt auch die Anzahl der Kontaktmöglichkeiten aus (Irrtumswahrscheinlichkeit von $5 \%$ ).

Wie schon im vorangegangenen Modell fällt auch hier der nicht signifikante Einfluss der Unternehmensgröße auf (Anzahl der Beschäftigten). Während die Betriebsgröße im Bereich der örtlichen Betriebsräte wohl zu den erklärungskräftigsten Variablen überhaupt gezählt werden muss, erfährt dieser Aspekt im Bereich der Gesamtbetriebsräte offenkundig einen fundamentalen Bedeutungswandel. Mehr noch als auf die Beschäftigtenzahl in der Einheit „Unternehmen“ als eher abstraktem Konzept kommt es wohl auf die konkrete Nähe und Erfahrbarkeit von „Belegschaft" im betrieblichen und damit oftmals auch im räumlichen Kontext an.

\section{Fazit und Diskussion}

An anderer Stelle (Behrens 2005b) hatten wir basierend auf der Befragung von örtlichen Betriebsräten bereits festgestellt, dass die Einbettung der Betriebsratsarbeit in komplexe Vertretungsnetzwerke (dort definiert als Verbindung von örtlichen Betriebsräten mit mindestens einer der folgenden Betriebsratsebenen: Gesamt-, Konzern- und Eurobetriebsrat) zwar deutliche Hinweise auf eine Professionalisierung der Betriebsratsarbeit liefert, aber keine Indizien für eine Entfremdung der Betriebsräte von den Gewerkschaften ergab. ${ }^{6}$ Folgt man der nun hier vorgenommenen Analyse, die auf einer deutlich ausgeweiteten Datenbasis beruht und über die Parallelbefragung der Gesamtbetriebsräte darüber hinaus eine zweite Perspektive einbezieht, so bestätigt sich dieser Befund insofern, als dass wir keine belastbaren Belege dafür vorgefunden haben, dass eine Zuständigkeitsverlagerung in Richtung Gesamtbetriebsrat zu einer Schwächung der Identifikation des örtlichen Betriebsrates mit der Gewerkschaft führt. Insofern kann - zumindest auf Ebene des örtlichen Betriebsrates - von einer Entfremdung des Betriebsrates von den Gewerkschaften nicht
6 Darüber hinaus ergab die Analyse schwache Hinweise auf die Formalisierung der Betriebsratsarbeit als Folge der Einbettung in komplexe Gremienstrukturen. 
die Rede sein. Auch auf der Ebene des Kontaktes zu den Belegschaften - Entfremdung in Form verminderte Präsenz in den Arbeitsbereichen - ergibt sich kein signifikanter Einfluss der Zuständigkeitsverteilung. Würde man die Analyse an dieser Stelle beenden, d.h. den Gesamtbetriebsratseinfluss ausschließlich über die abgeleitete Perspektive des örtlichen Betriebsrates thematisieren, könnte insofern „Entwarnung" gegeben werden, als dass eine Kompetenzverlagerung in Richtung Gesamtbetriebsrat die effektive Vertretung von Beschäftigteninteressen nicht beeinträchtigt.

Ein Blick auf die Analyseebene des Gesamtbetriebsrats verrät nun, dass dies vermutlich vorschnell wäre. So zeigen sich auf beiden Ebenen der Entfremdung (von der Gewerkschaft und von der Belegschaft) signifikante Koeffizienten für den Einfluss der Zuständigkeitsverteilung (die allerdings unterschiedliche Vorzeichen tragen). So ist die durch den Gesamtbetriebsrat wahrgenommene Identifikation mit den Gewerkschaften positiv mit einer Dezentralisie- rung der Kompetenzverteilung assoziiert. Bezogen auf unser Entkopplungsargument hat dies einige Konsequenzen. Zunächst einmal, dass der örtliche Betriebsrat in seiner eigenen Wahrnehmung weniger anfällig für die Erschütterung seines Bandes zu den Gewerkschaften ist als der Gesamtbetriebsrat. Selbst bei statistischer Kontrolle des Organisationsgrades und der Bewertung der gewerkschaftlichen Unterstützung finden wir in diesem Fall eine Entkoppelung des GBR von der organisierenden Gewerkschaft vor. Offensichtlich hat sich mit der Übernahme von Verantwortlichkeiten eine Reihe von GBR-Gremien zu einem autonomen Akteur der industriellen Beziehungen umdefiniert.

Anders verhält sich dieser Effekt im Bereich der Kontaktmöglichkeiten mit der Belegschaft, d.h. der Durchführung von Betriebsbegehungen. Mit der vermehrten Übernahme von Zuständigkeiten durch den GBR, also einem Prozess der Zentralisierung, steigt die Wahrscheinlichkeit, dass der Gesamtbetriebsrat Betriebsbegehungen durchführt. Ob dies Resultat einer Art
Kompensationsgeschäft ist, demzufolge mit der Übernahme von Kompetenzen gleichsam auch die Erklärungsbedürftigkeit direkt gegenüber der Belegschaft wächst, der GBR also „Flagge zeigen“ muss, kann hier nicht abschließend geklärt werden.

Letztlich bleiben, bezogen auf die Konsequenzen für die Interessenvertretungspraxis, ambivalente Resultate zu verbuchen. Die hier präsentierten Befunde deuten zwar nicht auf eine Loslösung der gewählten Vertretung von der Basis, also den Beschäftigten. Es ergeben sich aber durchaus Hinweise auf eine Schwächung der Identität des Verhältnisses zwischen Gesamtbetriebsräten und Gewerkschaften im dualen System. Ob dies letztlich ein Zeichen von Rollendifferenzierung ist, bei der sich einzelne Kollektivakteure auf ihre eigenen Vertretungsdomänen berufen, oder ein Zeichen von Entkoppelungsprozessen innerhalb des dualen Systems der Interessenvertretung, kann mit dieser Untersuchung nicht abschließend geklärt werden.

\section{LITERATUR}

Behrens, M. (2005a): Die Rolle der Betriebsräte bei der Werbung von Gewerkschaftsmitgliedern, in: WSI-Mitteilungen 6, S. 329-338 Behrens, M. (2005b): Die Arbeit des Betriebsrats in komplexen Gremienstrukturen, in: WSI-Mitteilungen 11, S. 638-644

Britz, G. (2006): Gesamt- und Konzernbetriebsrat: Nur zuständig, wenn unvermeidbar?, in: Die Personalvertretung 4, S. 131-132

Hoffmann, A. (2005): The Construction of Solidarity in a German Central Works Council: Implications for European Works Councils, unveröffentlichte Ph. D.-Dissertation, University of Warwick

Rancke, F. (1982): Betriebsverfassung und Unternehmenswirklichkeit. Eine Analyse von Organisation und Formen der betrieblichen Arbeitnehmermitbestimmung in der Praxis von Großunternehmen und Konzernen, Opladen
Schmidt, R. /Trinczek, R. (1991): Duales System: Tarifliche und betriebliche Interessenvertretung, in: Müller-Jentsch, W. (Hrsg): Konfliktpartnerschaft, 1. Auflage, München und Mering, S. 167-200

Schmidt, R./Trinczek, R. (1999): Der Betriebsrat als Akteur der industriellen Beziehungen, in: Müller-Jentsch, W. (Hrsg.): Konfliktpartnerschaft, 3. Auflage, München und Mering, S.103-128

Wassermann, W. (2002): Betriebsräte - Akteure für Demokratie in der Arbeitswelt, Münster

Wassermann, W./Rudolph, W. (2005): Betriebsräte nach der Reform. Eine empirische Untersuchung ausgewählter Effekte der Reform des Betriebsverfassungsgesetzes 2001 in der Praxis, Münster Weiss, M. (2006): The effectiveness of labour law: reflections based on the German experience, in: Managerial Law 3, S. 275-287 